\title{
Recent developments on the microstructural effects caused by small- charge explosions in FCC alloys
}

\author{
Donato FIRRAO ${ }^{1, a}$, Paolo MATTEIS ${ }^{1, b}$ and Chiara POZZI ${ }^{1, c}$ \\ ${ }^{1}$ Department of Material Science and Chemical Engineering, \\ Politecnico di Torino, Corso Duca degli Abruzzi 24, It-10129, Torino, Italy \\ adonato.firrao@polito.it, bpaolo.matteis@polito.it, 'chiara.pozzi@polito.it
}

Keywords: Twinning, Slip, FCC alloys, optical microscopy, X-Ray diffraction

\begin{abstract}
Metals exposed to small charge explosions, even in absence of overall deformation, show characteristic and permanent microstructural features, that can be related to blast wave properties, e.g. to the charge mass and the charge-to-target distance. In particular, Face Centered Cubic (FCC) alloys with low stacking fault energy may exhibit mechanical twinning due to the high strain rate caused by an explosion, even if in slower processes they mainly deform by slip. In some forensic science investigations, these crystallographic modifications, and particularly the occurrence of twinning, may be among the few remaining clues of a small charge explosion, and may be useful to hypothesize the nature and location of the charge. A wide experimental campaign was performed to correlate the blast wave properties with the ensuing modifications of FCC metal targets, and to investigate the microscopic deformation mechanisms leading to these modifications. In particular, it was attempted to identify the threshold conditions (charge-to-target distance, charge mass, and hence applied stress) that yield barely detectable microstructural modifications, and to study the transition from slip to twinning. FCC metal alloys, with low ( $\alpha$-brass, stainless steel), intermediate (copper, gold alloy), or high (aluminum alloy) stacking fault energy, were exposed to blast waves (caused by 50 or $100 \mathrm{~g}$ plastic explosive charges located at increasing charge-to-target distances) and then analyzed by X-ray diffraction, optical microscopy, scanning electron microscopy, and electron backscattered diffraction imaging. A comprehensive review of the most significant findings of the whole research, together with theoretical considerations on the slip and twinning deformation mechanisms, is here presented.
\end{abstract}

\section{Introduction}

Effects on metal targets after an explosion include fracture, macroscopic and localized plastic deformations, surface modifications and microstructural crystallographic alterations [1]. For small charge explosions, macroscopic effects are restricted to very small charge-to-target distances (of the order of tens of millimeters), whereas, at larger distances, most alterations are crystallographic. Moreover, in face centered cubic (FCC) alloys with low stacking fault energy $\left(\gamma_{s f}\right)$, a transition from slip to twinning can occur by increasing the deformation rate.

In some forensic science investigations, these crystallographic modifications, and particularly the occurrence of twinning, may be among the few remaining clues of a small explosion, and may be useful to hypothesize the nature and location of the charge. Thus, an experimental program has been undertaken to correlate the blast wave properties with the ensuing modifications observed in blast exposed FCC metal targets with low to high $\gamma_{s f}$ values.

Since the final goal was to investigate the modifications that can be induced in metal objects by an open field explosion, the chosen experimental setup is different from those usually encountered in shock loading experiments (e.g. those using a flier plate or a gas gun), in that the samples were subjected not only to the blast pressure wave, but also to its heat wave. Moreover, most of the literature, starting from the pioneer work by Smith [2], deals with very high shock pressures (e.g. from 10 to $10^{2} \mathrm{GPa}$ ), leading to clearly detectable twins, whereas almost no attention was devoted to 
shock pressures lower than $1 \mathrm{GPa}$, that could be characteristic of the transition between slip and twinning. Thus, the present study was aimed to single out the threshold conditions of detectability of crystallographic alterations, and to study the possible transition between slip and twinning.

Whereas most results were reported in previous papers [3-6], each addressing in detail one or two alloys, the present work presents a comprehensive review of the most significant findings.

\section{Experimental methods}

Disk shaped samples, with $20.5 \mathrm{~mm}$ diameter and $3 \mathrm{~mm}$ thickness, were either machined from wrought bars of AISI 304Cu stainless steel, 2014 aluminum alloy and of OFHC copper, or punched from sheets of $\alpha$-brass (30 wt.\% $\mathrm{Zn})$ and 18 carat gold alloy $(10 \% \mathrm{Ag}, 15 \% \mathrm{Cu})$. All tested materials, except the aluminum alloy, were annealed; the stainless steel and the gold alloy were annealed at two different temperatures, yielding slightly different grain sizes (Table 1); the aluminum alloy samples were solubilized and aged. The alloy compositions and heat treatments were reported in more detail elsewhere [3-6]. All disks were grinded, polished and etched before the explosions, so that the exposed surface could be analyzed as such after the test, avoiding the risk of introducing artifacts not related to the explosive event.

Spherical unconfined charges of plastic explosive (wt.\% composition: pentrite $86 \%$, plasticizer $11 \%$, polymer $2 \%$, carbon black $0.45 \%$ ) were exploded by cylindrical detonators inserted at their cores. The charges were hung from the detonator electrical cables and the samples' polished and etched surfaces were placed horizontally below them. To approximate a free-surface condition on the face not directly exposed to the shock wave, each sample was supported only by a thin circumferential rim of a wooden sample-holder; more details were given elsewhere [4]. Tests were performed using 50 or $100 \mathrm{~g}$ charges (TNT equivalent mass $m$ equal to 54.5 or $109 \mathrm{~g}$ [4]) and with different charge-to-sample distances, $d$, between the charge center and the sample upper surface (Table 1). The sample thickness was measured (with uncertainty $\pm 0.01 \mathrm{~mm}$ ) before and after the tests. The blast exposed surfaces were examined as-such; then the samples were cut in half by electrical discharge machining and the cross-sections were polished, etched and examined. Asprepared samples were similarly examined for comparison.

Table 1 - Overview of the parameters used for the explosions tests for the different materials.

\begin{tabular}{|c|c|c|c|c|c|c|c|}
\hline Alloy & $\begin{array}{c}\text { Grain size } \\
{[\mu \mathrm{m}]}\end{array}$ & $\begin{array}{c}m \\
{[\mathrm{~g}]}\end{array}$ & $\begin{array}{c}d \\
{[\mathrm{~mm}]}\end{array}$ & $\begin{array}{c}r \\
{\left[\mathrm{~kg} / \mathrm{m}^{1 / 3}\right]}\end{array}$ & $\begin{array}{c}P^{\mathrm{a}} \\
{[\mathrm{MPa}]}\end{array}$ & $\begin{array}{c}\tau_{\max }^{\mathrm{a}} \\
{[\mathrm{MPa}]}\end{array}$ & $\begin{array}{c}d_{e} \\
{[\mathrm{~mm}]}\end{array}$ \\
\hline \multicolumn{2}{|c|}{$\alpha$-brass } & 100 & $70-420$ & $0.15-0.88$ & $(98)-15$ & $(34)-5$ & 220 \\
\hline \multirow{2}{*}{$\begin{array}{c}\text { AISI 304Cu } \\
\text { stainless steel }\end{array}$} & 60 & 50 & $65-815$ & $0.17-2.15$ & $(83)-(6)$ & $(43)-(3)$ & 115 \\
\cline { 3 - 9 } & 30 & 100 & $120-420$ & $0.25-0.88$ & $(55)-15$ & $(29)-8$ & 170 \\
\hline \multirow{2}{*}{18 carat gold alloy } & 46 & 100 & $70-240$ & $0.15-0.50$ & $(98)-27$ & $(19)-5$ & 170 \\
\cline { 2 - 8 } & 55 & 100 & $70-320$ & $0.15-0.67$ & $(98)-20$ & $(19)-4$ & 170 \\
\hline \multicolumn{2}{|c|}{ OFHC Cu } & 100 & $70-200$ & $0.15-0.42$ & $(98)-33$ & $(34)^{\mathrm{b}}-11$ & 110 \\
\hline \multicolumn{2}{|c|}{ AA 2014 } & 50 & $65-135$ & $0.17-0.36$ & $(83)-39$ & $(29)^{\mathrm{b}}-14$ & 135 \\
\hline
\end{tabular}

${ }^{\mathrm{a}}$ From experimental values, values in brackets are extrapolated $\quad{ }^{\mathrm{b}}$ Invalid due to plastic deformation

\section{Shock Wave Properties}

For dimensional reasons, the blast waves properties depend on the reduced distance, defined as $r=$ $d \cdot m^{-1 / 3}[8,10]$. The peak overpressure exercised on the sample's exposed surface, $P$, as well as the overpressure rise time, were estimated either from instrumented explosion tests performed in the 0.3 
to $0.88 \mathrm{~m} \cdot \mathrm{kg}^{-1 / 3}$ reduced distance range [4], or from previous literature data concerning the same reduced distance range [9], by considering the pressure increase due to the reflection of the blast wave on the sample [10]; the examined $r$ and $P$ ranges are given in Table 1.

In the case of tests at charge-to-target distances close to, or larger than, the microstructural variation detectability thresholds, and causing limited or nil final plastic deformation, the sample stress history can be estimated by using the dynamic elasticity theory. The overpressure rise times are in all cases much longer than the time needed for a shock wave to travel twice through the sample. It follows that the stress history of any point inside the sample consisted of only one significant pressure rise and pressure decrease (plus negligible oscillations), with an overall maximum value that decreases with the distance from the exposed surface; no relevant tensile stress occurs [4]. Thus, the maximum shear stress $\tau_{\max }$ occurs at the most favorable orientation at the exposed surface and can be calculated according to Meyers [11]; the examined $\tau_{\max }$ ranges are listed in Table 1 .

\section{Results}

Macroscopic deformation. The disks thickness was not modified by the explosions, except for samples very close to the charges, which showed either a slight decrease in thickness due to compression, or a slight increase owing to melting and resolidification of large areas of the surface. Increasing the charge-to-target distance, these effects vanish, and the theory of elasticity can be considered valid. Thus, the shear stress $\tau_{\max }$ calculated with the abovementioned hypotheses is considered valid for distances larger than the distances $d_{e}$ listed in table 1 .

Microstructures. Original microstructures, as observed by Optical Microscopy (OM), were fully recrystallized with equi-axial polygonal grains and frequent annealing twins. Only the aluminum alloy samples did not exhibit annealing twins, due to the high $\gamma_{s f}$ [12], and showed regions either of equi-axial grains or of smaller grains oriented in the plastic deformation direction of the original wrought bar (normal to the surface then exposed to the explosions).

The modifications of the exposed surfaces, observed after the explosions by Optical Microscopy $(\mathrm{OM})$ and Scanning Electronic Microscopy (SEM), include: melting and resolidification, either complete or partial (at grain boundaries or upon limited areas); impact craters with deposition of oxidized explosive components and of material originated from detonator case fragments, and series of crystal plastic deformation parallel marks limited by the grain boundaries.

The melting and resolidification phenomenon occurred in most samples exposed to the explosions at the smallest distances. In most cases it was inhomogeneous and the areas not affected showed the other features discussed here, especially the parallel crystal plastic deformation marks.

The crystal plastic deformation parallel marks (Fig. 1) were evident in regions where, apparently, the original grain boundaries and annealing twin boundaries had not been altered by the blast waves. The marks were always limited by the grain boundaries and limited or deviated by the annealing twin boundaries. These marks were more frequent and evident around the fragment impact points, and were observed at these particular points even in samples subjected to explosions at the largest distances, in which they were elsewhere absent.

The modifications observed by OM in the cross-sections were generally restricted to similar series of parallel crystal plastic deformation marks, occurring in a thin layer close to the exposed surface.

The parallel marks are planarity variations of the original polished and etched surface, directly due to the shock wave; they are generally interpreted as the traces of either mechanical twin planes, or slip planes (a cross-slip behavior can be hypothesized in some instances), or combinations of both.

At the lower reduced distances, the parallel marks occupy the whole exposed surface (except the melting and resolidification areas); by increasing the reduced distance, the parallel marks are gradually less evident and finally either limited to areas around fragment impact points or very rare, short, shallow and restricted to a few isolated grains. The surface detectability thresholds, reported in Table 2 for each alloy, are defined as the maximum charge-to-target distance (or minimum overpressure or shear stress) at which these surface deformation marks were still observed in all 
grains (in the unmelted areas). In the aluminum alloy case, all tested samples showed surface deformation marks; thus, tests at larger distances are needed to determine the surface threshold.

Since from OM analysis it is not possible to distinguish between slip and twin traces, analyses with Scanning Electron Microscopy (SEM) coupled with Electron Backscattered Diffraction (EBSD) were planned. A first scan on $\alpha$-brass did not show different crystal orientations in areas with parallel deformation marks, but was not conclusive due to a relatively large step size (about 10 $\mu \mathrm{m} / \mathrm{step})[6]$. Further scans on a gold sample, performed with a very small step size (100 $\mathrm{nm})$ evidenced the parallel marks as narrow strips with a crystal orientation different from that of their parent grain [13]. Further EBSD analyses are ongoing.

Table 2 - Threshold distances and stresses for the detection of crystal deformation surface marks. Extreme values at which the surface marks were observed / not observed.

\begin{tabular}{|c|c|c|c|c|c|}
\hline Alloy & $\begin{array}{c}\text { Grain size } \\
{[\mu \mathrm{m}]}\end{array}$ & $\begin{array}{c}m \\
{[\mathrm{~g}]}\end{array}$ & $\begin{array}{c}d \\
{[\mathrm{~mm}]}\end{array}$ & $\begin{array}{c}r \\
{\left[\mathrm{~kg} / \mathrm{m}^{1 / 3}\right]}\end{array}$ & $\begin{array}{c}\tau_{\max } \\
{[\mathrm{MPa}]}\end{array}$ \\
\hline$\alpha$-brass & & 100 & $270 / 320$ & $0.57 / 0.67$ & $8.4 / 7.1$ \\
\hline \multirow{2}{*}{$\begin{array}{c}\text { AISI 304Cu } \\
\text { stainless steel }\end{array}$} & 60 & 50 & $195 / 215$ & $0.51 / 0.57$ & $13.7 / 12.4$ \\
\cline { 3 - 7 } & 30 & 100 & $240 / 270$ & $0.50 / 0.57$ & $14 / 12.4$ \\
\hline \multirow{2}{*}{18 carat gold alloy } & 46 & 100 & $240 / 270$ & $0.50 / 0.57$ & $14 / 12.4$ \\
\cline { 2 - 6 } & 55 & 100 & $220 / 320$ & $0.46 / 0.67$ & $5.7^{\S} / 3.9$ \\
\hline \multicolumn{2}{|c|}{ OFHC Cu } & 100 & $90 / 110$ & $0.19 / 0.23$ & $26 / 21$ \\
\hline \multicolumn{2}{|c|}{ AA 2014 } & 50 & $135 /$ n.a. & $0.35 /$ n.a. & $14 /$ n.a. \\
\hline \multicolumn{2}{|c}{ Invalid due to plastic deformation or surface melting } \\
\hline
\end{tabular}

\section{Discussion and conclusions}

For most FCC alloys, at low strain rate the critical shear stress for slip, $\tau_{\mathrm{c}, \mathrm{sl}}$, is lower than the critical shear stress for twinning, $\tau_{\mathrm{c}, \mathrm{tw}}$, but increases with the deformation rate. Conversely, $\tau_{\mathrm{c}, \mathrm{tw}}$ is almost constant with the strain rate, and is an increasing function of the stacking fault energy, $\gamma_{s f}$. Thus twinning can occur at high strain rates in FCC alloys with low $\gamma_{s f}$ as a single deformation mechanism, or alongside slip, depending on the strain rate and on the maximum shear stress.

The $\gamma_{s f}$ of the metals and alloys treated here, estimated from the literature, are $15 \mathrm{~mJ} / \mathrm{m}^{2}$ for $\alpha$-brass [17], $31 \pm 8 \mathrm{~mJ} / \mathrm{m}^{2}$ for AISI $304 \mathrm{Cu}$ [15], $78 \mathrm{~mJ} / \mathrm{m}^{2}$ for pure copper [16], and $166 \mathrm{~mJ} / \mathrm{m}^{2}$ for pure aluminum [16]. On this bases, and neglecting the influence of alloying elements in the case of the aluminum alloy, the relationship by Firrao et al. [1] yields a $\tau_{\text {c,tw }}$ value of $33 \mathrm{MPa}$ for $\alpha$-brass, 69 MPa for stainless steel, $177 \mathrm{MPa}$ for pure copper and $367 \mathrm{MPa}$ for the aluminum alloy. Finally, for a silver-gold alloy with $75 \mathrm{wt} . \%$ gold, a $\tau_{\mathrm{c}, \mathrm{tw}}$ of $92.3 \mathrm{MPa}$ was proposed by Suzuki and Barret [18].

On the other hand, typical values of critical shear stress for slip at low deformation rates, $\tau_{\mathrm{c}, \mathrm{s}}$, vary from $10^{-4}$ to $10^{-5} G$, where $G$ is the shear modulus [19], giving values in the order of magnitude of $10^{-1}$ to $10^{1} \mathrm{MPa}$ for the examined metals and alloys.

From the present results and hypotheses, for all the analyzed materials, the shear stress detectability threshold, $\tau_{\mathrm{th}}$, is much lower than the estimated twinning stress $\tau_{\mathrm{c}, \mathrm{tw}}$, suggesting that the observed deformation marks, at least in the vicinity of the thresholds, are due mainly to slip. Nevertheless, in the case of $\alpha$-brass, the critical twinning stress was exceeded for charge-to-target distances below $120 \mathrm{~mm}$, and the XRD patterns suggested, for the charge-to target distances between 140 and 220 $\mathrm{mm}$, the occurrence of twinning phenomena, even if the estimated shear stress $\tau_{\max }$ was lower than $\tau_{\mathrm{c}, \mathrm{tw}}$ in this range. A similar result is observed also in the case of stainless steel, where mechanical 
twins were observed [3], and this was explained by hypothesizing the influence of other effects (e.g. constrained thermal dilatation) alongside the blast pressure.

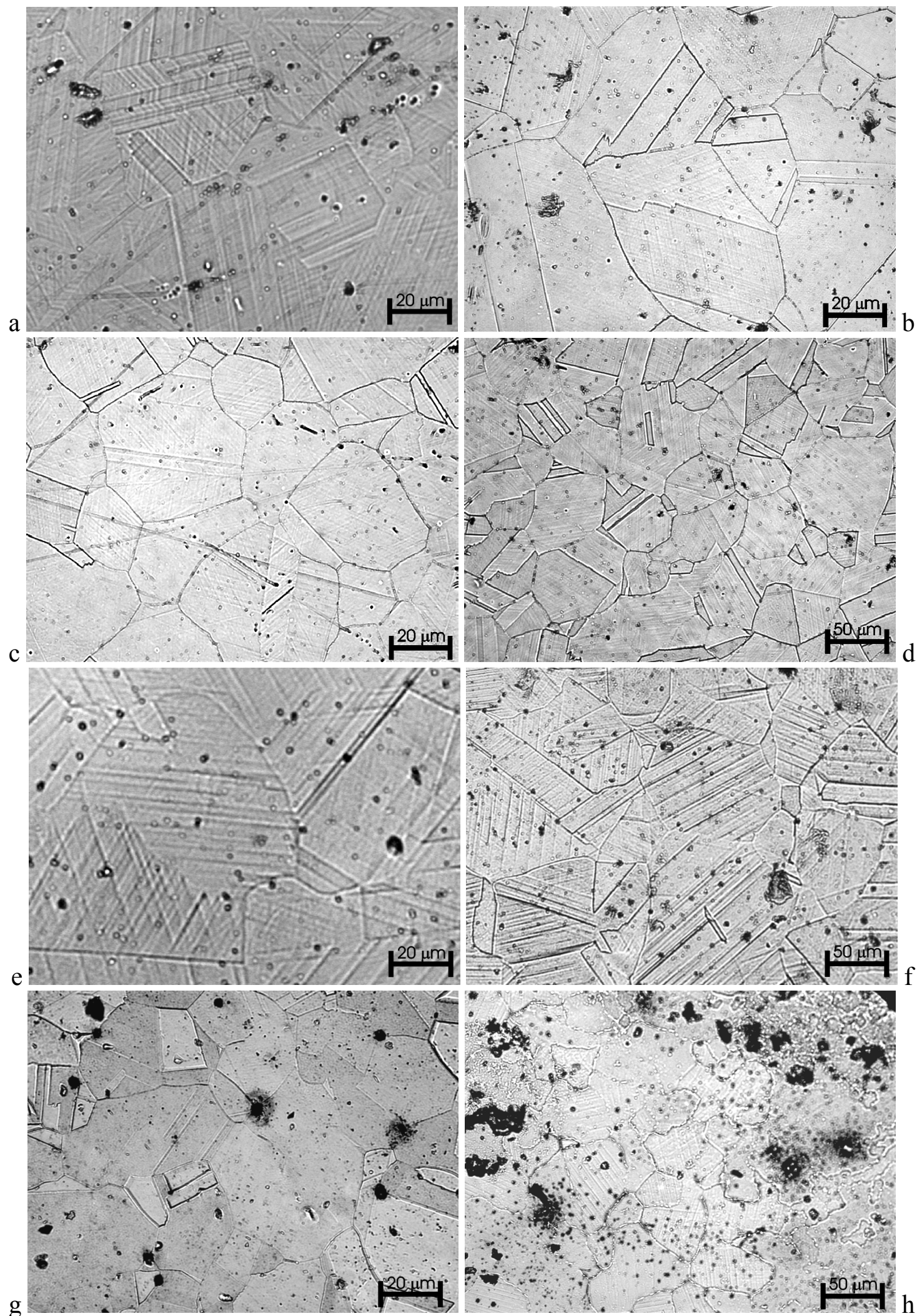

Fig. 1 - Surfaces exposed to the blast wave at reduced distances close to $0.35 \mathrm{~m} / \mathrm{kg}^{1 / 3}$. Deformation marks in all grains in $\alpha$-brass (a, $0.29 \mathrm{~m} \mathrm{~kg}^{-1 / 3}$ ), AISI $304 \mathrm{Cu}$ stainless steel (b, $60 \mu \mathrm{m}$ grain size and $50 \mathrm{~g}$ charge mass; c, $60 \mu \mathrm{m}, 100 \mathrm{~g} ; \mathrm{d}, 30 \mu \mathrm{m}, 100 \mathrm{~g}$ ) and 18 carat gold alloy (e, $46 \mu \mathrm{m}$; f, $50 \mu \mathrm{m}$ ), almost no effects in pure copper (g), melted areas and deformation marks in AA 2014 (h). 
In the stainless steel case, mechanical twins were detected and threshold distances defined, allowing to develop a criterion to identify unknown explosive events. In the case of $\alpha$-brass, the plastic deformation marks can be attributed to slip at the largest distances, and to combined slip and twinning effects at the shortest distances, on the basis of the estimated shear stress thresholds for the two phenomena. As it regards gold alloy and pure copper, the deformation marks are due mainly to slip, even if the presence of some twins in the most favorably oriented directions cannot be excluded. In the case of the examined aluminum alloy, the occurrence of twinning can be ruled out. The ongoing SEM/EBSD analyses are expected to be conclusive in highlighting the relative contribution of the two deformation mechanisms. Nevertheless, being these marks slip or twins, metallographic analyses can yield useful information about the occurrence of an explosive event, if the production process is known and extensive slips caused by the latter process can be excluded.

\section{Acknowledgements}

The authors wish to thank their colleagues from University of Genova, University of Roma Tor Vergata, and Italian Navy who gave their contribution to the present research [3-6], as well as the Italian Ministry of University and Research for financial support by grant no. 200109974.

\section{References}

[1] D. Firrao, G. Ubertalli, E. Cordano, Tech. Law Ins. 4 (1999), p. 23.

[2] C.S. Smith, Trans. AIME 212 (1958), p. 574.

[3] D. Firrao et al., Mater. Sci. Eng. A 424 (1) (2006), 23.

[4] D. Firrao et al., Metall. Mater. Trans. A 38A (2007), p. 2869.

[5] D. Firrao et al., TMS 2008 Annual Meeting Supplemental Proceedings, Vol. I: Materials Processing and Properties, TMS (USA), 1, (2008), p. 327.

[6] D. Firrao et al., Calphad, in press.

[7] R.W. Armstrong, P.J. Worthington, In Metallurgical Effects At High Strain Rates, R.W. Rhode, J.R. Holland and C.K. Harnes Eds. Plenum Press, New York, NY, (1964), p-401-414.

[8] H.L. Brode, J. App. Phys. 26 (1955), p. 766.

[9] J. Henrych, The Dynamic of Explosion and its Use, Elsevier, Amsterdam, Holland, 1979.

[10] S. Petralia, Compendio di esplosivistica, Mariperman, La Spezia, Italy, 2000.

[11] M.A. Meyers, K.K. Chawla, Mechanical Metallurgy: Principles and Applications, PrenticeHall, Englewood Cliffs, NJ, (1984), 536.

[12] L.E. Murr, Acta Metall, 21, (1973), p. 791

[13] J. F. Bingert, C. Pozzi, Unpublished results, LANL, Los Alamos, NM, USA (2009)

[14] B.E. Warren, X-Ray Diffraction, Addison Wesley, Reading, MA, USA, 1969, p. 275.

[15] D. Firrao, P. Matteis, C. Pozzi, Acc. Sci. Torino-Mem. Sci. Fis. Ser., 29 (2005), p. 3.

[16] L.E. Murr, Interfacial Phenomena in Metals and Alloys, Addison Wesley, Reading, MA, USA, (1975), p. 145-147.

[17] A. Howie, P.R. Swann, Phil. Mag., 6 (1961), p.1215.

[18] H. Suzuki, C. Barrett, Acta Metall. 6 (1958), p. 156.

[19] R.W. Cahn, P. Haasen (Eds.), Physical Metallurgy, North-Holland, Amsterdam, Holland, 1996. 